\title{
Risk factors for onset of delirium after neck of femur fracture surgery: a prospective observational study
}

\author{
Muaaz Tahir ${ }^{1, *}$, Shahbaz S. Malik ${ }^{2}$, Usman Ahmed ${ }^{1}$, Jakub Kozdryk ${ }^{1}$, Syeda Huma Naqvi ${ }^{2}$, and Atul Malik ${ }^{2}$ \\ 1 The Royal Orthopaedic Hospital NHS Foundation Trust, Birmingham, UK \\ 2 Sandwell and West Birmingham Hospitals NHS Trust, Birmingham, UK
}

Received 7 November 2017, Accepted 19 April 2018, Published online 6 July 2018

\begin{abstract}
Background: Delirium is a common complication after surgery in the elderly that leads to increased length of stay and other adverse outcomes. The aim of this study was to better understand the exact causes of post-operative delirium in patients undergoing surgery for neck of femur (NOF) fractures.

Methods: We performed a prospective cohort study of 381 consecutive patients undergoing surgery for NOF fractures at a single institution. Baseline cognitive status and risk factors were recorded on admission. Postoperative cognitive status was assessed at regular intervals until discharge. Binary logistic regression was performed to identify independent predictors of delirium.

Results: Patients who developed post-operative delirium $(n=70)$ were significantly older (average age 83 vs. 78 , $p=0.019)$ and more likely to be female $(79 \%$ vs. $67 \%, p=0.062)$ than non-affected patients. The presence of delirium was associated with increased length of stay (13 vs. 10 days, $p=0.001)$ and 1-year mortality ( $25.7 \%$ vs. $15 \% p=0.03$ ). Independent predictors of delirium included age $\geq 65$ years (Odds Ratio $=5.8$ ), presence of anaemia $(\mathrm{OR}=2.9)$, hypoxia $(\mathrm{OR}=2.86)$, cardiac disease $(\mathrm{OR}=2.8)$, Chronic Obstructive Pulmonary Disease $(\mathrm{OR}=2.5)$, new onset electrolyte imbalance $(\mathrm{OR}=2.2)$ and renal failure $(\mathrm{OR}=1.9)$.

Conclusion: Overall analysis demonstrated an increased incidence of delirium in older females with greater comorbid conditions. It was also found to be associated with increased morbidity and mortality. We recommend clinicians put greater effort into recognising risk factors of delirium and diagnosing it in a timely manner to mitigate its effects.
\end{abstract}

Key words: Neck of femur fracture, Rehabilitation, Delirium, Risk factors, Post-operative complications.

\section{Introduction}

Postoperative delirium is a common complication of surgical interventions in the elderly. Characterised by acute and fluctuating impairment of cognition, attention, and consciousness, it is a multifactorial condition that is associated with poor outcomes. Although its incidence is substantially influenced by patient-related risk factors, previous reviews have indicated that it affects a large proportion of orthopaedic patients. In 2007, a metaanalysis of 26 studies reported higher rates of delirium in patients undergoing hip fracture surgery (ranging from 4 to 53 percent) compared with elective surgery (3.6-28.3 percent) [1]. Delirium has immediate adverse implications for the orthopaedic patient such as an increased risk of major complications, slow recovery and therefore a longer inpatient stay. Patients are typically less compliant with rehabilitation protocols and are more likely to require nursing home placement after discharge. In the longer

\footnotetext{
*Corresponding author: Muaaz.tahir@nhs . net
}

term, it is also associated with the development of dementia [2]. As a preventable condition in 30-40\% of cases [3] delirium holds substantial public health relevance as a target for interventions to prevent its associated burden of downstream complications and costs [4].

Causes of postoperative delirium in patients with hip fracture are complex and not well understood [5]. Its onset is thought to reflect an accumulation of predisposing patient risk factors and the physiological stress of surgery [6]. Thus, identifying at risk individuals is critical to creating prevention and treatment pathways. The purpose of this prospective observational study was to determine the commonest risk factors for new onset delirium after hip fracture surgery in the UK, to establish its incidence, and associated mortality.

\section{Materials and methods}

In this single-center, prospective, cohort study we enrolled all patients presenting to our institution with a neck of femur (NOF) fracture between 2015 and 2016. Our 
Table 1. Diagnostic criteria for delirium according to DSM-IV [7].

A Disturbance of consciousness (i.e. reduced clarity of awareness of the environment) with reduced ability to focus, sustain, or shift attention.

B A change in cognition (such as memory deficit, disorientation, language disturbance) or the development of a perceptual disturbance that is not better accounted for by a pre-existing, established, or evolving dementia.

C The disturbance develops over a short period of time (usually hours to days) and tends to fluctuate during course of the day.

D There is evidence from the history, physical examination, or laboratory findings that the disturbance is caused by the direct physiological consequences of a general medical condition.

primary objective was to evaluate the incidence and predisposing risk factors for developing delirium after NOF fracture surgery. We compared patients with newonset post-operative delirium to those who did not develop delirium using multiple variables at each stage of their treatment pathway. For purpose of analysis the following data were extracted: patient's age and gender; date of injury; co-morbidities; place of residence before admission; cognitive function at the time of admission; time to surgery; type of anaesthesia and procedure performed. Other variables studied in the postoperative period were presence of anaemia $(\mathrm{Hb}<100 \mathrm{~g} / \mathrm{L})$, new onset hypoxaemia, significant hypotension, renal failure, and date of death if applicable. Patient records were reviewed at 1year to assess rate of mortality.

\section{Setting}

Our institution is a busy acute hospital with 470 beds serving a population of around 290000 . There is a wellestablished NOF fracture treatment pathway which aims for prompt haemodynamic resuscitation, early clinical assessment, radiological diagnosis and definitive management within $36 \mathrm{~h}$ of attendance. Patients are offered prompt and appropriate analgesia (which may include an ilio-fasical block in addition to oral analgesics) in the emergency department. Patients are then admitted to a dedicated orthopaedic ward for pre-operative optimisation, surgical and orthogeriatric assessment. Surgery is performed in line with national guidance preferring cemented hemiarthroplasty (or total hip arthroplasty) in patients with a displaced intracapsular fracture. Extracapsular fractures are treated using dynamic hip screw fixation or cephalomedullary devices in most cases. Where appropriate, patients are offered a choice of spinal or general anaesthesia after assessment by the consultant anaesthesist. Postoperative rehabilitation protocols were similar in all patients. Patients were routinely seen by the physiotherapists and occupational therapists as of the first postoperative day. Discharge destination post-surgery varies from rehabilitation wards within the trust, intermediate care centres to home with a safe package of care. The study was approved by our institutional review board.

\section{Diagnosis of delirium}

A ten-point abbreviated mental test (AMT) assessment was performed at the time of admission to assess baseline cognitive function. Patients were then assessed
Table 2. Patient demographics.

\begin{tabular}{llll}
\hline & Non-delirious & Delirious & $p$ \\
\hline Total number of patients & $311(82 \%)$ & $70(18 \%)$ & - \\
Average age (median year) & 78 & 83 & 0.019 \\
$<25$ years & $3(100 \%)$ & 0 & - \\
25-45 years & $5(100 \%)$ & 0 & - \\
$46-64$ years & $37(95 \%)$ & $2(5 \%)$ & - \\
65-74 years & $42(83 \%)$ & $9(17 \%)$ & - \\
$\geq 75$ years & $224(79 \%)$ & $59(21 \%)$ & - \\
Sex (\% female) & 67.2 & 78.6 & 0.062 \\
Nottingham hip fracture & $5[3-6]$ & $5[5-6]$ & 0.071 \\
score (median [IQR]) & & & \\
Pre-existing dementia & $115(37 \%)$ & $36(51 \%)$ & 0.026 \\
\hline
\end{tabular}

daily on ward rounds by a senior orthogeriatric physician. Any change in consciousness and/or cognition reported in the physician or nursing notes was recorded. Presence of delirium was confirmed using the Diagnostic and Statistical Manual of Mental Disorders (DSM 4th Edition) criteria (Table 1) [7].

\section{Data analysis}

Statistical analysis was performed using SPSS version 20 (IBM; Armonk, NY). Significant differences between groups were identified by independent $t$ tests for continuous variables and $\chi^{2}$ tests for categorical variables. Binary logistic regression using the stepwise method was performed to determine independent risk factors for postoperative delirium. $p$ values $<0.05$ were considered statistically significant.

\section{Results}

During the 12-month study period, 395 patients underwent surgery for NOF fracture at our institution. Fourteen patients were excluded from analysis due to missing data. Of the remaining 381 patients, 70 patients (18\%) developed new-onset delirium post-operatively. Patients experiencing delirium were significantly older (average age 83 vs. $78, p=0.019$ ) and more likely to be female $(79 \%$ vs. $67 \%, \quad p=0.062)$ than non-affected patients (Table 2). Patients who already had a diagnosis of dementia on admission were significantly more likely to 
Table 3. Treatment modality.

\begin{tabular}{|c|c|c|c|}
\hline & Non-delirious $(n)$ & Delirious $(n)$ & $p$ \\
\hline Dynamic hip screw & $152(82 \%)$ & $33(18 \%)$ & $\overline{0.793}$ \\
\hline Hemiarthroplasty & $91(76 \%)$ & $28(24 \%)$ & 0.08 \\
\hline Total Hip Replacement & $20(95 \%)$ & $1(5 \%)$ & 0.098 \\
\hline Intramedullary Nail & $45(87 \%)$ & $7(13 \%)$ & 0.325 \\
\hline
\end{tabular}

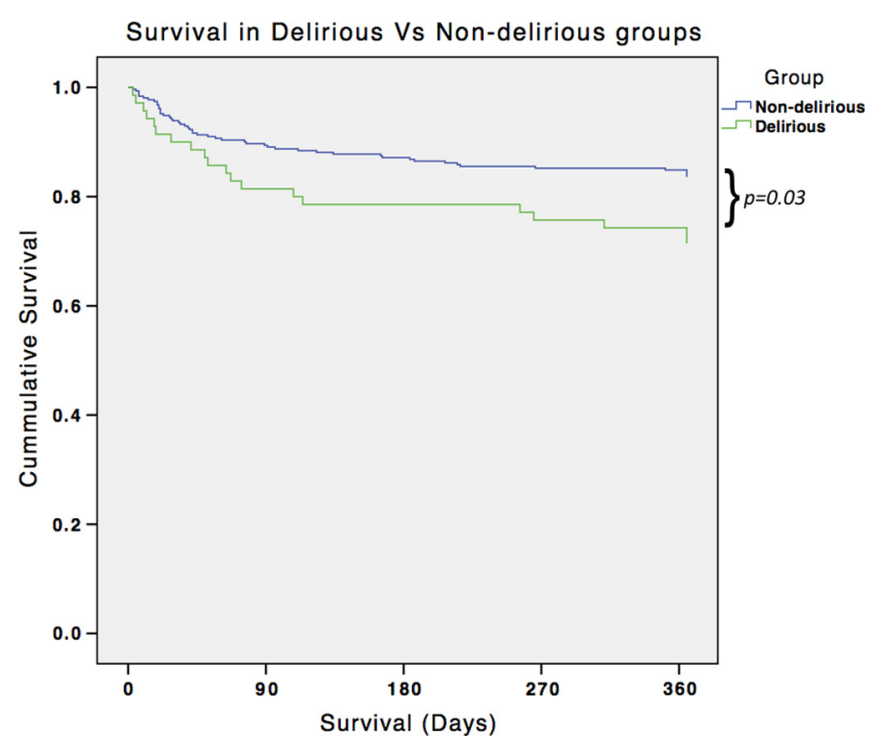

Figure 1. Kaplan Meier plot.

develop post-operative delirium compared with those with no cognitive dysfunction on admission (51\% vs. $37 \%$ respectively, $p=0.02$ ).

The majority of patients were treated with either a dynamic hip screw (47\%) or a hemiarthroplasty (30\%) with no significant differences in choice of treatment between the two groups (Table 3 ).

Presence of delirium was associated with significantly increased length of stay (average Length of stay 13 days vs. 10 days, $p=0.001$. Incidence of mortality at 1 year was significantly higher in patients with a diagnosis of postoperative delirium $(25.7 \%)$ compared to patients without delirium (15\%) $p=0.026$ (Figure 1). Mortality within 30-days followed the same trend (10\% vs. $6 \%)$ however did not reach statistical significance $(p=0.243)$ (Table 4).

Logistic regression analysis identified 7 independent predictors for postoperative delirium (Table 5). These included age $\geq 65$ years (OR 5.8, CI 1.4-24.3), presence of anaemia (OR 2.9, CI 1.5-5.5), new onset hypoxia (OR 2.86, CI 1.4-5.8), cardiac disease (OR 2.8, CI 1.5-5), COPD (OR 2.5, CI 1.5-4.2), new onset electrolyte imbalance (OR 2.2 CI 1.2-3.7) and renal failure (OR 1.9, CI 1.1-3.3). Factors which did not show statistically significant association with the development of delirium included delay in surgery i.e. $>36 \mathrm{~h}$ from admission to surgery (OR 0.98, CI 0.5-1.8), use of general anaesthetic
Table 4. Mortality.

\begin{tabular}{rllc}
\hline & Non-delirious & Delirious & $p$ \\
\hline 30 days & $6 \%$ & $10 \%$ & 0.24 \\
1 year & $15 \%$ & $25.7 \%$ & 0.03 \\
\hline
\end{tabular}

Table 5. Risk factors for postoperative delirium.

\begin{tabular}{|c|c|c|c|c|}
\hline \multirow[b]{2}{*}{ Age $\geq 65$} & \multirow{2}{*}{$\frac{\begin{array}{l}\text { Odds } \\
\text { ratio }\end{array}}{5.75}$} & \multicolumn{2}{|c|}{$\begin{array}{l}95 \% \text { confidence } \\
\text { interval }\end{array}$} & \multirow{2}{*}{$\begin{array}{c}p \\
0.017\end{array}$} \\
\hline & & 1.36 & 24.3 & \\
\hline $\mathrm{Hb}<100$ & 2.91 & 1.53 & 5.54 & $<0.001$ \\
\hline New onset hypoxaemia & 2.86 & 1.4 & 5.84 & 0.004 \\
\hline Cardiac disease & 2.76 & 1.52 & 5.04 & 0.001 \\
\hline Renal failure & 1.92 & 1.13 & 3.27 & 0.016 \\
\hline COPD & 2.47 & 1.46 & 4.18 & 0.001 \\
\hline $\begin{array}{l}\text { New onset electrolyte } \\
\text { imbalance }\end{array}$ & 2.15 & 1.23 & 3.75 & 0.007 \\
\hline $\begin{array}{l}>36 \mathrm{~h} \text { from admission } \\
\text { to surgery }\end{array}$ & 0.98 & 0.53 & 1.82 & 0.95 \\
\hline Use of general anaesthetic & 1.18 & 0.53 & 2.64 & 0.692 \\
\hline
\end{tabular}

(OR 1.2, CI 0.5-2.6), prolonged operating time i.e. $>2 \mathrm{~h}$ (OR 1.3, CI 0.6-2.7), and post-operative hypotension i.e. $>30 \%$ from baseline (OR 0.9, CI 0.4-2.0).

\section{Discussion}

Neck of femur fractures are a major public health concern due to the increasing ageing population. Majority of fractures occur in patients over the age of 65 , frequently in those with frailty and dependency, and with preexisting medical problems. For these reasons, it provides a good clinical model to study and understand delirium. This study focused on incident (or new onset) delirium which occurred exclusively in the postoperative period. We identified a number of independent risk factors that correlate with the onset of post-operative delirium in NOF fracture patients: increasing age; post-operative anaemia; cardiorespiratory co-morbidities, and acute electrolyte imbalance. Identifying patients at increased risk of delirium after surgery could enable pre-emptive action to mitigate this risk.

Since the implementation of National guidelines on hip fracture management and severe financial penalties for healthcare trusts that fail to comply with efficiency targets, most institutions, including ours, have welldefined treatment pathways to facilitate early surgery and targeted rehabilitation [8,9]. Moreover, orthogeriatrician-led perioperative care has led to a notable reduction in morbidity and mortality amongst hip fracture patients. In our study, we found a much lower incidence of delirium than that found in previous studies [10-12]. This finding may in part be explained by the aforementioned improvements in care but there are several other possible explanations including variations in definition of delirium 
and diagnostic accuracy. Because delirium is by nature a fluctuant disorder, which may sometimes last for a short duration or in its hypoactive form, it is possible that some cases may have been missed.

Previous reports have associated post-operative delirium with increased length of hospital stay, rate of discharge to a nursing home, health and social care costs $[13,14]$. Similar findings were observed in this study. Presence of delirium on average amounted to 3 extra days of hospital stay. Although cost analysis was beyond the scope of our study, based on recently published figures, it is estimated that a district general hospital in the NHS would incur an additional $£ 894$ per patient for 3 days of inpatient orthopaedic or rehabilitative care [15]. This estimate excludes the cost of any additional physiotherapy or occupational therapy sessions, additional nursing procedures or ward rounds, and the cost of treating delirium itself. Finally, adding on costs for potential post discharge dependency, such as nursing home care, the financial burden of postoperative delirium can rise even higher.

Controversy exists regarding whether postoperative delirium is an independent predictor of mortality [16,17]. In our study, about one-quarter of patients who developed delirium died within 1 year, which was significantly higher than non-delirious patients. However, given the high prevalence of coexisting frailty and morbidity in these patients, a direct causal relationship cannot be determined. Indeed, dementia, COPD, chest infection, heart failure, anaemia, electrolyte disturbance, elevated urea/creatinine, and malignancy, have all been described as risk factors for increased mortality in the months following a hip fracture [18]. An important limitation of our study was that prospective data on patients' health and function was only recorded until the time of discharge. Other factors that may have contributed to mortalities that occurred following discharge from hospital, remain largely unknown.

Delirium occurs as a consequence of a combination of predisposing, non-modifiable factors - such as age, coexisting medical illnesses etc. - and precipitating factors which are often modifiable. The risk factors identified through logistic regression in our study are generally in agreement with previous reports on postoperative delirium. Elderly patients are considered to be at higher risk because many of the aforementioned predisposing risk factors accumulate and overlap with ageing. For this reason, delirium was most frequently observed amongst the octogenarians. However, through binary logistic regression analysis, patients as young as 65 years of age were found to have a significantly increased risk of developing delirium compared to those younger than 65 years. Although predictors such as age are unmodifiable, they should be used in stratifying high risk patients on admission so that preventative strategies can be better targeted towards those at high risk. Modifiable risk factors such as anaemia and hypoxia can be prevented or treated to avoid prolonged cerebral hypoxaemia, which is thought to be an important underlying phenomenon in the development of delirium $[19,20]$. A number of previous studies investigating the role of anaemia and blood transfusion within the prevention and treatment strategies for delirium have also found low hemoglobin level to be associated with delirium, and receiving a blood transfusion to be associated with a lower delirium incidence [21]. Similarly, supplemental oxygen (3-4L/ min) continually till day 2 post-surgery, or while patient's oxygen saturation is not $\geq 95 \%$ without oxygen, have proven to reduce delirium risk [22,23]. Based on previous systematic reviews, the Association of Anaesthetists of Great Britain and Ireland (AAGBI) and Scottish Intercollegiate Guidelines Network (SIGN) recommend the use of regional anaesthesia with the aim of avoiding general anaesthesia and opioid drugs in all patients unless contraindicated $[7,8,24]$. In this study, general anaesthesia was not associated with an increased risk of developing post-operative delirium. A more up-to-date systematic review is currently underway evaluating both experimental and observational evidence on the use of regional compared to general anaesthesia in patients undergoing hip fracture surgery, with a focus on delirium as an outcome [25]. Because delirium is considered a multifactorial condition, addressing a single risk factor is unlikely to be successful, therefore multicomponent approaches will be most effective for both prevention and treatment.

Despite significant improvements in both surgery and rehabilitation in recent decades, hip fracture remains, for patients and their carers, a much-feared injury. Because the onset of delirium signals underlying ill health, a comprehensive multidisciplinary approach is required in treatment and indeed prevention of delirium. A clear understanding of risk factors for postoperative delirium helps in the selection of individuals who might benefit from targeted perioperative intervention.

\section{Conflict of interest}

The authors declare that they have no conflict of interest.

\section{References}

1. Bruce AJ, Ritchie CW, Blizard R, Lai R, Raven P (2007) The incidence of delirium associated with orthopedic surgery: a meta-analytic review. Int Psychogeriatr 19(2), 197-214.

2. Saczynski JS1, Marcantonio ER, Quach L, Fong TG, Gross A, Inouye SK, Jones RN (2012) Cognitive trajectories after postoperative delirium. N Engl J Med 367(1), 30-39.

3. Marcantonio ER, Flacker JM, Wright RJ, Resnick NM (2001) Reducing delirium after hip fracture: a randomized trial. J Am Geriatr Soc 49(5), 516-522.

4. O'Mahony R, Murthy L, Akunne A, Young J (2011) Synopsis of the national institute for health and clinical excellence guideline for prevention of delirium. Ann Intern Med 154(11), 746-751.

5. Brauer C, Morrison RS, Silberzweig SB, Siu AL (2000) The cause of delirium in patients with hip fracture. Arch Intern Med 160(12), 1856-1860. 
6. American Geriatrics Society Expert Panel on Postoperative Delirium in Older Adults. Postoperative Delirium in Older Adults: best practice statement from the American Geriatrics Society. J Am College Surg 220(2), 136-148.

7. American Psychiatric Association. Diagnostic and Statistical Manual of Mental Disorders, 4th Ed. (DSM-IV). Washington, DC, APA 1994.

8. Griffiths R, Alper J, Beckingsale A, et al. (2012) Management of proximal femoral fractures 2011: association of anaesthetists of Great Britain and Ireland. Anaesthesia $67(1), 85-98$.

9. Scottish Intercollegiate Guidelines Network. Edinburgh: SIGN Guidline 111; 2009.

10. Marsland D, Colvin PL, Mears SC, Kates SL (2010) How to optimize patients for geriatric fracture surgery. Osteoporos Int 21, S535-S546.

11. Vidán M, Serra JA, Moreno C, Riquelme G, Ortiz J (2005) Efficacy of a comprehensive geriatric intervention in older patients hospitalized for hip fracture: a randomized, controlled trial. J Am Geriatr Soc 53, 1476-1482.

12. Shields L, Henderson V, Caslake R (2017) Comprehensive geriatric assessment for prevention of delirium after hip fracture: a systematic review of randomized controlled trials. J Am Geriatr Soc 65(7), 1559-1565.

13. Holmes J, House A (2000) Psychiatric illness predicts poor outcome after surgery for hip fracture: a prospective cohort study. Psychol Med 30, 921-929.

14. McCusker J, Cole MG, Dendukuri N, et al. (2003) Does delirium increase hospital stay? J Am Geriatr Soc 51, 1539-1546.

15. Yasin MN, Saqib R (2015) Trauma healthcare treatment costs in the national health service. Bull Royal Coll Surg Engl 97(1), 28-31.

16. Nightingale S, Holmes J, Mason J, et al. (2001) Psychiatric illness and mortality after hip fracture. Lancet 357, 12641265 .
17. Hamilton GM, Wheeler K, Di Michele J, et al. (2017) A systematic review and meta-analysis examining the impact of incident postoperative delirium on mortality. Anesthesiology $127(1), 78-88$.

18. Carpintero P, Caeiro JR, Carpintero R, Morales A, Silva S, Mesa M (2014) Complications of hip fractures: a review. World J Orthop 5(4), 402-411.

19. Rolfson D. (2002) The causes of delirium. In: Delirium in old age. Lindesay J, Rockwood K, Macdonald A, eds. Oxford, Oxford University Press, 101-122.

20. Burns A, Gallagley A, Byrne J (2004) Delirium. J Neurol Neurosurg Psychiatry 75, 362-367.

21. van der Zanden V, Beishuizen SJ, Scholtens RM (2016) The effects of blood transfusion on delirium incidence. J Am Med Dir Assoc 17(8), 748-753.

22. Dovjak P, Iglseder B, Mikosch P, Gosch M, Müller E, Pinter G, Pils K, Gerstofer I, Thaler H, Zmaritz M, WeissenbergerLeduc M, Müller W (2013) Treatment and prevention of postoperative complications in hip fracture patients: infections and delirium. Wien Med Wochenschr 63, 448454.

23. Lundström M, Olofsson B, Stenvall M, Karlsson S, Nyberg L, Englund U, Borssén B, Svensson O, Gustafson Y (2007) Postoperative delirium in old patients with femoral neck fracture: a randomized intervention study. Aging Clin Exp Res 19(3), 178-186.

24. Papaioannou A, Fraidakis O, Michaloudis D, Balalis C, Askitopoulou H (2005) The impact of the type of anaesthesia on cognitive status and delirium during the first postoperative days in elderly patients. Eur J Anaesthesiol 22, 492-499.

25. Yeung J, Patel V, Champaneria R, Dretzke J (2016) Regional versus general anaesthesia in elderly patients undergoing surgery for hip fracture: protocol for a systematic review. Syst Rev 5, 66 .

Cite this article as: Tahir M, Malik SS, Ahmed U, Kozdryk J, Naqvi SH, Malik A (2018) Risk factors for onset of delirium after neck of femur fracture surgery: a prospective observational study. SICOT-J, 4, 27. 